\section{Financiamiento de la salud mental en Chile: una deuda pendiente}

\author{
PAULA ERRÁZURIZ ${ }^{1,2, a}$, CAMILA VALDÉS ${ }^{1, b}$, \\ PAUL A. VÖHRINGER ${ }^{3,4, c}$, ESTEBAN CALVO ${ }^{5, \mathrm{~d}}$
}

\section{Mental health financing in Chile: a pending debt}

In spite of the high prevalence of mental health disorders in Chile, there is a significant financing deficit in this area when compared to the world's average. The financing for mental health has not increased in accordance with the objectives proposed in the 2000 Chilean National Mental Health and Psychiatry Plan, and only three of the six mental health priorities proposed by this plan have secure financial coverage. The National Health Strategy for the Fulfilment of Health Objectives for the decade 2011-2020 acknowledges that mental disorders worsen the quality of life, increase the risk of physical illness, and have a substantial economic cost for the country. Thus, this article focuses on the importance of investing in mental health, the cost of not doing so, and the need for local mental health research. The article discusses how the United States is trying to eliminate the financial discrimination suffered by patients with mental health disorders, and concludes with public policy recommendations for Chile.

(Rev Med Chile 2015; 143: 1179-1186)

Key words: Chile; Government financing; Mental health; Parity; Public health.
'Escuela de Psicología, Pontificia Universidad Catolica de Chile,

Santiago, Chile.

${ }^{2}$ Centro Nacional de Investigación para la Gestión Integrada de Desastres

Naturales (CIGIDEN), Santiago, Chile.

${ }^{3}$ Clínica Psiquiátrica Universitaria,

Hospital Clínico Universidad de Chile,

Facultad Medicina Universidad de

Chile, Santiago, Chile.

${ }^{4}$ Mood Disorders Program, Tufts

Medical Center, Tufts University School

of Medicine, Boston, USA.

Instituto de Políticas Públicas,

Universidad Diego Portales, Santiago,

Chile.

aPsicóloga y Ph.D. en Psicología Clínica.

bicenciada en Ciencias Biológicas y

Candidata a Doctor en Psicología.

'M.Sc. Investigación Clínica, M.P.H.

¿Sociólogo, Ph.D. en Sociología y M.Sc. en Salud Pública.

Fuente de apoyo financiero: CONICYT/ FONDAP/Nº15110017.

Recibido el 30 de noviembre de 2013, aceptado el 28 de julio de 2015.

Correspondencia a:

Paula Errázuriz Arellano

Escuela de Psicología, Pontificia

Universidad Catolica de Chile.

Avda. Vicuña Mackenna 4860, Macul,

Santiago.

Fono: 2354 1245; Fax: 23544844

paulae@uc.cl

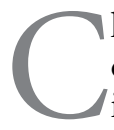

hile es un país con altas tasas de enfermedades mentales, cuyos costos directos e indirectos afectan negativamente a los individuos que las padecen, sus familias y la sociedad en su conjunto, aumentando -por ejemplo- los costos y usos de los servicios generales de salud. Si bien el Ministerio de Salud ha reconocido crecientemente la relevancia de los problemas de salud mental, el financiamiento de la salud mental no ha aumentado según los objetivos propuestos. Los planes de salud mental implementados en la última década no han reducido las altas tasas de enfermedades mentales.

Las enfermedades psiquiátricas son un factor de riesgo para la conducta suicida ${ }^{1,2}$. A nivel mundial, la Organización Mundial de la Salud estimó en 1998 que al suicidio correspondería a 1,8\% de la carga mundial de enfermedades, esperando que aumente a 2,4\% para el año $2020^{1}$. Chile es el segundo país de la OCDE que más ha aumentado su tasa de suicidios durante los últimos 15 años $^{3,4}$. Además, durante la última década el suicidio ha sido una de las diez primeras causas de muerte en varones chilenos ${ }^{5}$, concentrando $11,7 \%$ de las defunciones en población general y $19,1 \%$ de la mortalidad masculina en 2010. Hoy en día, la prevalencia de conducta suicida en la población chilena es mayor que en Nueva Zelanda y Japón, países donde el fenómeno es reconocidamente relevante $^{6}$. A su vez, en Chile el suicidio masculino toma más vidas anualmente que el SIDA y las enfermedades cardiovasculares ${ }^{7,8}$.

La presencia de trastornos mentales a largo plazo se ha asociado con una importante disminución en la calidad de vida, problemas de salud física y una alta tasa de mortalidad prematura ${ }^{9-11}$. Por ejemplo, la depresión y esquizofrenia aumentan entre 40 y $60 \%$ la probabilidad de sufrir 
una muerte prematura por suicidio o problemas de salud física, tales como cáncer, enfermedades cardio-vasculares, diabetes, o infección por $\mathrm{VIH}^{12}$. Los trastornos depresivos unipolares son la segunda causa de años de vida saludables perdidos por vivir con discapacidad (AVISA) en la población chilena, y la primera en la población femenina entre 20 y 44 años $^{13}$. Como se observa en la Figura 1, en su conjunto, las condiciones neuropsiquiátricas (trastornos depresivos unipolares y bipolares, esquizofrenia, dependencia y consumo alcohol y drogas ilícitas, trastornos ansiosos, epilepsia, demencias, enfermedad de Parkinson, migraña, parálisis cerebral infantil y síndrome de déficit atencional del niño) son el subgrupo de enfermedades que más AVISA concentra, abarcando $23,2 \%{ }^{13}$, y ocupando el primer lugar en gasto por licencias curativas ${ }^{14}$.

En este artículo se contribuye a la literatura previa e ilustra con datos nacionales e internacionales la importancia de invertir en salud mental, el costo de no hacerlo, y la necesidad de producir investigación nacional ${ }^{15}$. Se presenta el caso de Estados Unidos de Norteamérica como ejemplo del intento por acabar con la inequidad en el financiamiento y acceso a la salud mental, y se proponen recomendaciones para Chile.

\section{Políticas de salud mental en Chile}

El actual sistema de salud mental en Chile se basa en el Plan Nacional de Salud Mental y Psiquiatría del año 2000, el cual plantea múltiples propuestas sobre el financiamiento y enfermedades prioritarias, enfatizando un modelo comunitario en el tratamiento y prevención de enfermedades mentales ${ }^{16}$. En éste plan se definieron seis áreas prioritarias: 1) trastorno por déficit de atención e hiperactividad en niños; 2 ) víctimas de violencia intrafamiliar; 3) abuso y dependencia de alcohol y drogas; 4) depresión; 5) esquizofrenia, y 6) enfermedad de Alzheimer y otras demencias en adultos mayores. Actualmente tres de estas seis áreas (depresión, esquizofrenia, abuso y dependencia de sustancias) son cubiertas por AUGE/GES, plan que establece garantías de acceso, oportunidad, protección financiera, calidad de la atención entregada y entrega de los medicamentos garantizados ${ }^{17}$.

De las 80 patologías que actualmente cubre AUGE/GES, cuatro (5\%) corresponden a patologías neuropsiquiátricas, las que constituyen casi un cuarto de la carga total de enfermedades en Chile (AVISA) ${ }^{13}$. De las 10 patologías agregadas en 2010, y 11 agregadas en 2013 al plan AUGE/

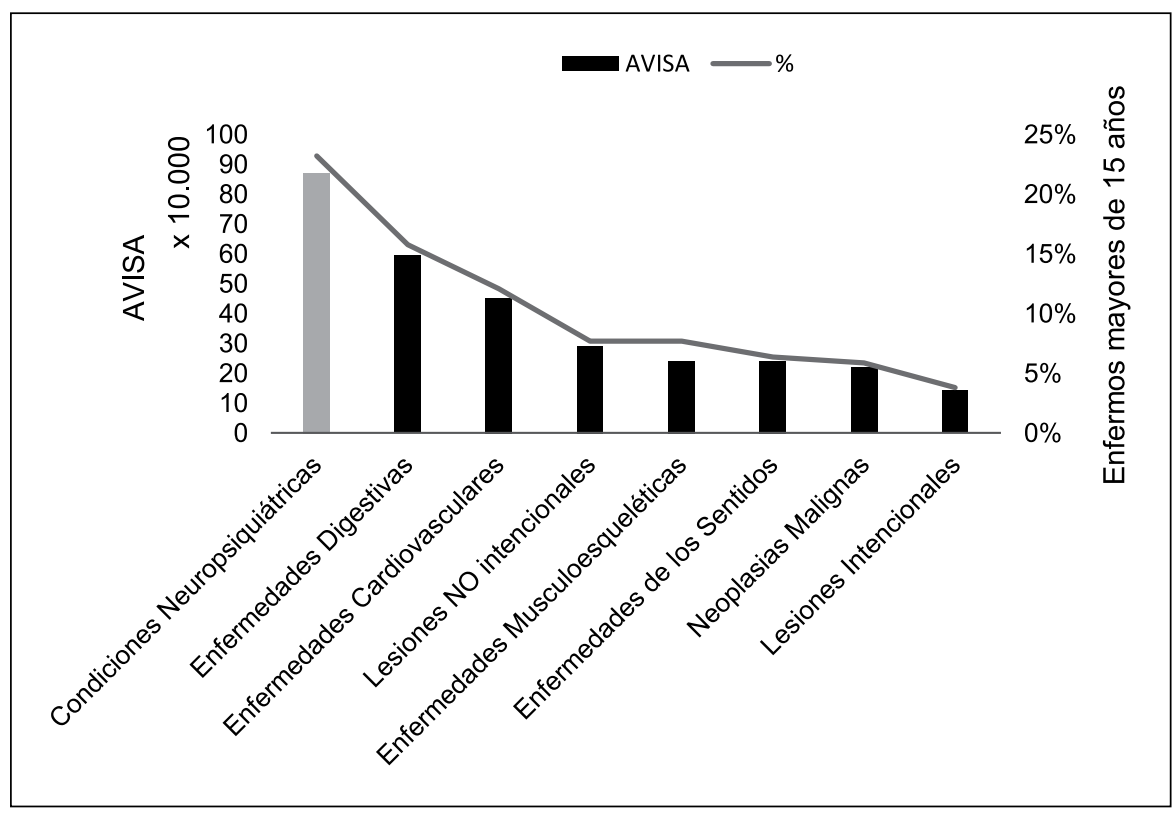

Figura 1. Años de Vida Ajustados por Discapacidad (AVISA) según subgrupo de enfermedades. AVISA: Suma de los años de vida perdidos en Chile $\%$ : Porcentaje de chilenos sobre 15 años que padecen alguna de las siguientes condiciones o enfermedades. 
GES, el trastorno bipolar es la única patología que corresponde al área de salud mental, quedando fuera el déficit atencional, la depresión adolescente y el maltrato infantil, las cuales estaban dentro las patologías a evaluar para ser incorporadas ${ }^{17}$. Si bien no se cuenta con estudios de prevalencia actualizados a la fecha, existen enfermedades altamente prevalentes -como la agorafobia, que afecta a $11 \%$ de los chilenos y es el trastorno mental más frecuente en Chile ${ }^{18,19}$ que tampoco han sido incorporadas al AUGE/ GES. Según Inostroza (2013), el aumento de patologías cubiertas por el AUGE/GES no ha sido acompañado de los recursos necesarios para cubrirlas $^{20}$, por lo que incluir más trastornos de salud mental en este plan tampoco asegura su adecuado financiamiento.

En 2011 el Gobierno de Chile presentó la Estrategia Nacional de Salud para el Cumplimiento de los Objetivos Sanitarios de la Década 20112020, conocida como "Elige Vivir Sano". En este documento se reconoce que las enfermedades mentales empeoran la calidad de vida, aumentan el riesgo de enfermedades físicas y tienen un costo económico muy alto para el país. En ella se proponen 50 objetivos de salud, de los cuales cuatro refieren directamente a metas de salud mental: 1) disminuir la prevalencia de discapacidad en personas con enfermedad mental; 2) reducir el consumo y riesgo asociado al alcohol; 3) disminuir el consumo de drogas ilícitas; y 4) disminuir la mortalidad adolescente asociada a suicidios ${ }^{21}$. La estrategia busca seguir el ejemplo de países como Inglaterra y Australia que han posicionado la temática de salud mental como una prioridad de política pública ${ }^{22,23}$.

\section{Financiamiento de la salud mental en Chile}

Según el informe de la Organización Mundial de la Salud y el Ministerio de Salud de Chile ${ }^{24}$, el año 2007 el sector público destinó 2,14\% del presupuesto total de salud a salud mental. En el año 2012, el porcentaje del presupuesto de salud que el sector público destinó a salud mental fue de $2,16 \%{ }^{25}$, cifra que está muy debajo de 5,0\% que propuso como meta el Plan Nacional de Salud Mental y Psiquiatría para el año $2010^{24}$ (Figura 2). Comparado con otros países de ingreso alto y medio alto, el porcentaje del presupuesto asignado a salud mental en Chile también parece relativamente bajo. Como se puede ver en la Figura 3, el porcentaje destinado a salud mental sitúa a nuestro país muy por debajo de los porcentajes que muestran otros países de ingresos altos como Estados Unidos de Norteamérica (6,0\%), Australia $(9,6 \%)$, Reino Unido (10,0\%), Suecia y Nueva Zelanda $(11,0 \%)$, y al promedio mundial $(2,8 \%)^{24}$.

En suma, a pesar de los esfuerzos de quienes prestan servicios de salud mental, las condiciones de financiamiento son insuficientes para alcanzar

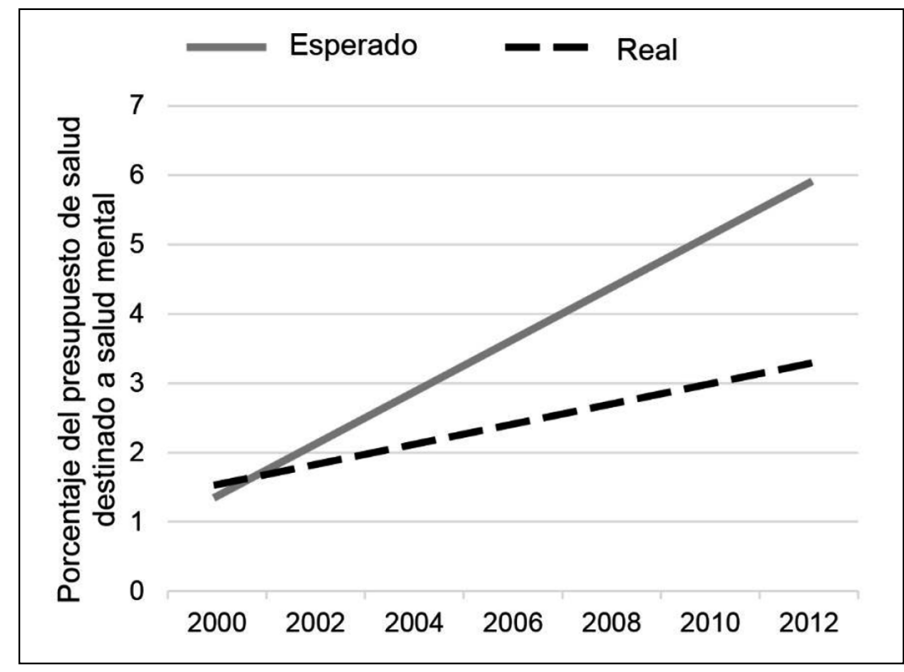

Figura 2. Porcentaje real del presupuesto de salud destinado a salud mental versus el esperado. 


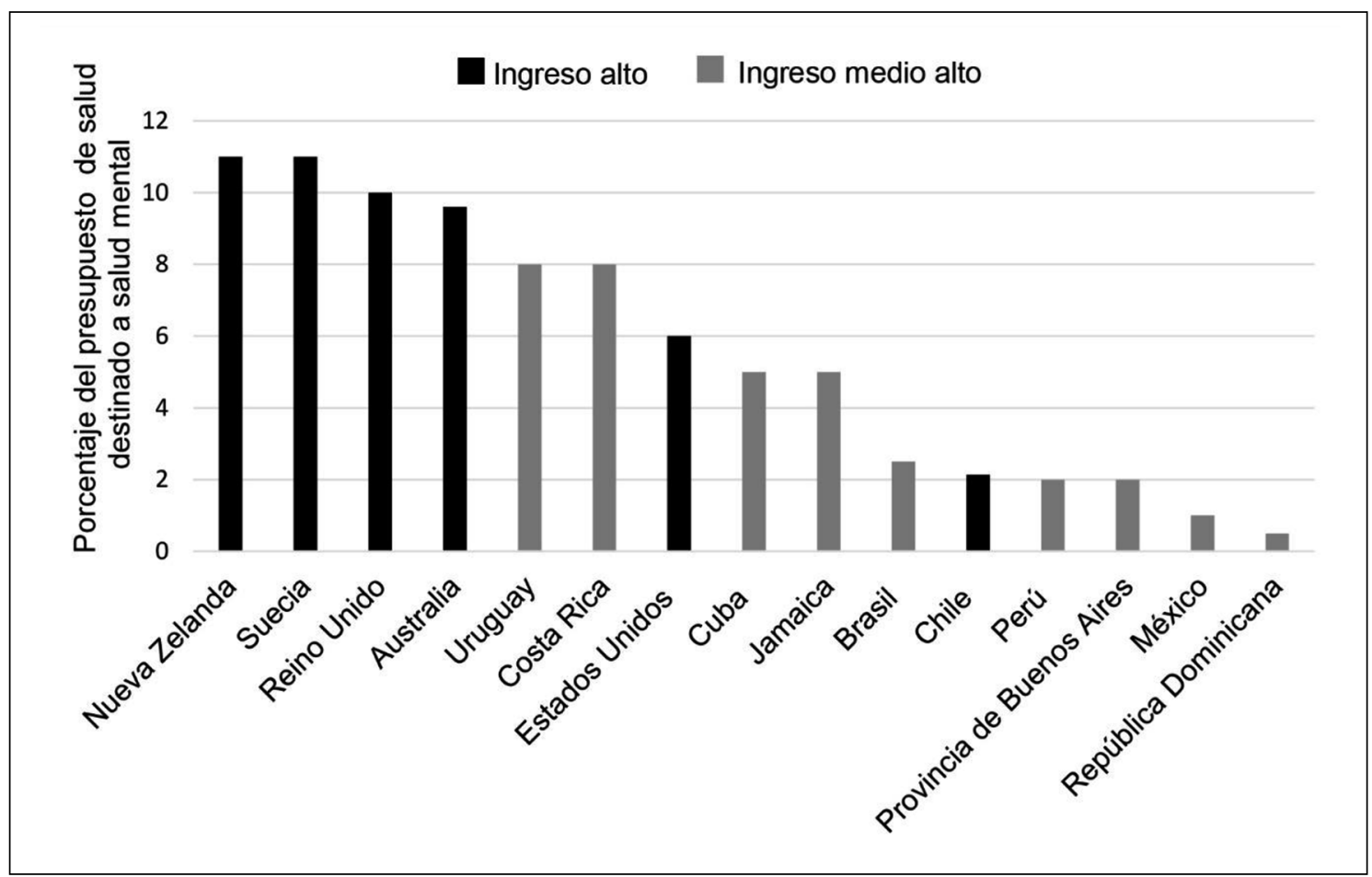

Figura 3. Porcentaje del presupuesto nacional de salud destinado a salud mental en países de ingreso alto y medio alto.

los objetivos trazados en materia de promoción, prevención y tratamiento de los trastornos mentales en Chile ${ }^{24}$. La falta de recursos económicos podría ser una de las razones que explican por qué el Plan Nacional de Salud Mental y Psiquiatría y la incorporación de patologías mentales al sistema AUGE/GES no han generado grandes cambios en la prevalencia de patología mental en Chile. Por ejemplo, si bien la depresión fue incorporada dentro de las patologías AUGE/GES en 2006, la presencia de síntomas depresivos en la población no ha disminuido de forma importante ${ }^{26,27}$. Mientras $17,5 \%$ de la población había presentado síntomas depresivos en el último año en 2003, esta cifra sólo disminuyó a $17,2 \%$ en 2009 , aumentando específicamente la prevalencia en mujeres de edad media ( 45 a 64 años) desde $22,2 \%$ a $30,1 \%$ y en los hombres de 25 a 44 años desde $8,3 \%$ a 11,0\%.

Respecto al suicidio, entre 2000 y 2009 las tasas aumentaron considerablemente (Figura 4). En 2000 hubo 9,6 fallecimientos por suicidios por cada 100.000 habitantes (16,6 en hombres y 2,7 en mujeres), subiendo a 12,7 en 2009 (20,8 en hombres y 5,0 en mujeres ${ }^{28}$. No sabemos cómo serían las tasas actuales de depresión y suicidio si la depresión no hubiera sido incorporada al AUGE/GES, pero es razonable especular que el panorama actual sería más sombrío aún, ya que se ha demostrado que el tratamiento de la depresión disminuye la prevalencia del suicidio ${ }^{29}$.

Los escasos estudios nacionales sobre prevalencia de otras patologías mentales, y sobre el impacto de las políticas de salud mental implementadas, no permiten tener un panorama más completo sobre la evolución de la salud mental en el país y la efectividad de los recursos invertidos. La mayoría de las investigaciones en salud mental centra su atención en los países del hemisferio norte ${ }^{30}$. Si bien constituyen antecedentes relevantes para la política pública nacional, las diferencias socioeconómicas y culturales limitan la generalización de los hallazgos. Por esto, además de destinar mayores recursos a financiar la salud mental, también es necesario contar con investigación nacional que permita impulsar intervenciones pertinentes, eficientes y costo-efectivas. 


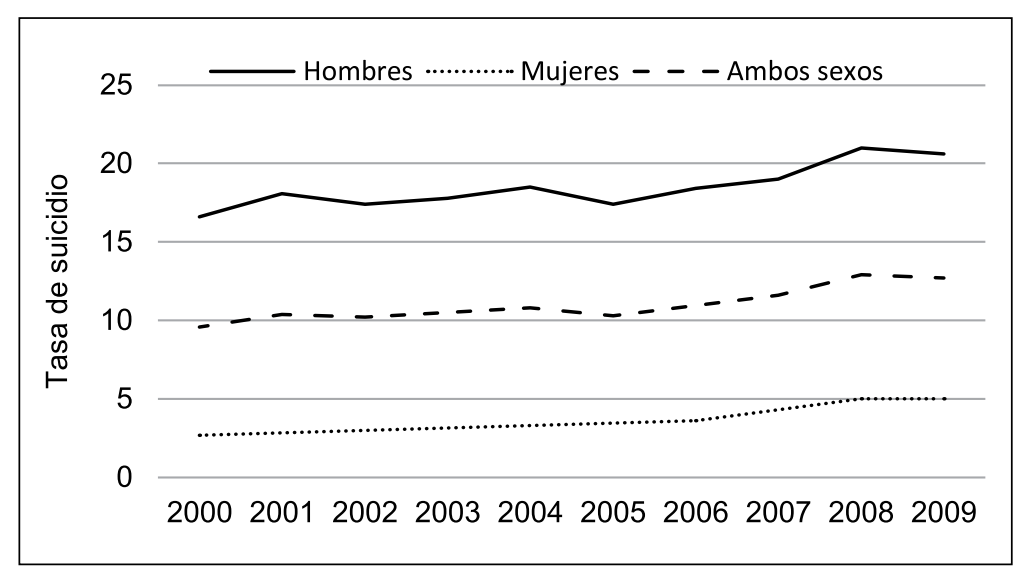

Figura 4. Cambio en la tasa de suicidio por cada 100.000 habitantes (2000-2009).

\section{El costo de no invertir en salud mental}

Si bien existe una alta prevalencia de trastornos de salud mental en Chile, y hasta la fecha el país no ha destinado los recursos necesarios para enfrentar esta situación, podría argumentarse que la salud mental no debería ser una prioridad nacional y que los individuos y sus familias deberían encargarse de este problema. Sin embargo, las enfermedades mentales imponen altos costos económicos tanto para las personas como para el país. Por ejemplo, la depresión tiene un mayor peso económico en el costo total de manejo de la enfermedad que el cáncer ${ }^{31}$. A diferencia de otras condiciones médicas, como las enfermedades cardiacas o la diabetes, los costos indirectos asociados a las enfermedades de salud mental y abuso de sustancias generalmente igualan o exceden los costos directos del tratamiento".

Los costos indirectos asociados a las enfermedades mentales incluyen el gasto gubernamental en vivienda, bienestar, educación y justicia, además de los costos vinculados a la pérdida de productividad de las personas ${ }^{32,33}$. Considerando diversos países, la tasa de ausentismo laboral por problemas de salud mental se ha estimado entre $35 \%$ y $45 \%{ }^{10}$. En Chile, los trastornos mentales y del comportamiento ocupan el primer lugar en el gasto por licencias curativas, concentrando $19,3 \%$, del gasto total por subsidio de cargo de las Isapres ${ }^{14}$. Con $18,2 \%$, estos trastornos representan el segundo mayor gasto en licencias médicas tramitadas. De éstas, 53,8\% son por episodios depresivos, $21,8 \%$ por trastornos de ansiedad y $16,8 \%$ por estrés grave y trastornos de adaptación. En general, la mayor prevalencia de sintomatología depresiva ocurre precisamente en la población en edad laboral ${ }^{34}$.

Los costos sociales vinculados a las enfermedades mentales también son altos. Los trastornos de salud mental están asociados al abuso de sustancias y a la pobreza. Ya que sufrir de un trastorno mental implica pérdida de productividad y el costo de un tratamiento a largo plazo, esto contribuye a mantener una situación de pobreza; al mismo tiempo, vivir en situación de pobreza contribuye a la aparición y mantención de los trastornos mentales, generando un círculo vicioso ${ }^{10}$. La depresión es 1,5 a 2 veces más frecuente en personas de nivel socioeconómico bajo que en personas de nivel socioeconómico alto, siendo la pobreza un factor que contribuyente significativamente al desarrollo de enfermedades mentales ${ }^{10,12,35}$. Es más, la Organización Mundial de la Salud considera que sin una inversión adecuada en salud mental se perpetua el círculo de la pobreza y se detiene el desarrollo ${ }^{10}$.

Las enfermedades mentales aumentan el uso y costos de los servicios generales de salud ${ }^{10}$. Tanto las personas portadoras de condiciones médicas crónicas como las que presentan trastornos mentales o abuso de sustancias no tratadas tienen más complicaciones y deben asumir mayores costos en tratamientos médicos. Las enfermedades mentales generan importantes impedimentos para el autocuidado y para la adherencia y respuesta a tratamientos de otras enfermedades. En particular, la depresión se presenta en $25 \%$ de los pacientes que consultan en atención primaria ${ }^{35}$. Esta enfermedad 
aumenta en $60 \%$ la probabilidad de sufrir diabetes tipo $2^{36}$, incrementa significativamente el riesgo de padecer enfermedad de Alzheimer ${ }^{37}$ y triplica el riesgo de morir de una enfermedad cardiaca ${ }^{38}$. Las personas con depresión severa reportan cuatro veces más dolor de cuello y espalda que aquéllos sin depresión o con depresión leve. En el caso de quienes sufren de diabetes, el tener depresión aumenta en 4,5 veces los costos de salud. Asimismo, aquellas personas que tienen tanto depresión como dolor de espalda faltan el doble al trabajo y duplican los costos médicos en comparación con quienes tienen sólo uno de estos diagnósticos ${ }^{36-39}$.

La eficacia del tratamiento de enfermedades mentales y abuso de sustancias ha sido demostrada empíricamente durante las últimas décadas. Cada vez más la evidencia económica indica que existen intervenciones para los trastornos mentales, tales como la esquizofrenia o la depresión, que son eficaces, asequibles y rentables ${ }^{10}$. El tratamiento adecuado de las patologías psiquiátricas mejora la salud general de las personas, disminuye los días de trabajo perdidos y la incapacidad laboral ${ }^{40}$. A su vez, los tratamientos de salud mental reducen la necesidad de hospitalizaciones y atención de emergencia, y mejoran los indicadores de salud de las personas con diabetes, cáncer, enfermedades cardiacas, dolor crónico y otras enfermedades graves $^{39,40}$. También se ha demostrado que las inversiones dirigidas a promover, prevenir, detectar y tratar tempranamente las enfermedades mentales pueden ser rentable y eficaces, produciendo retornos de la inversión inicial a mediano plazo, auto-financiamiento y disminución de los costos tanto en salud como en otras áreas (e.g. criminalidad), la mayoría en un plazo menor a 5 años $^{41,42}$. El desarrollo de programas de prevención y promoción de la salud mental dentro de las estrategias generales de los modelos de salud pública ayuda a evitar muertes, reducir el estigma asociado a las personas con trastornos mentales y mejora el entorno social y económico de un país ${ }^{10}$.

\section{Enfermedades físicas y mentales: un problema de equidad}

El bajo porcentaje del presupuesto total de salud que es destinado a salud mental no sólo preocupa porque los recursos son insuficientes para prevenir y tratar las patologías mentales, sino que además es una señal de la discriminación que viven las personas con trastornos mentales.

En Chile existe una diferencia importante en las posibilidades que tienen las personas de tratar sus enfermedades físicas y mentales. Éstas últimas han tenido históricamente una menor cobertura por parte de las distintas instancias de financiamiento, ya sean públicas o privadas, lo que implica una discriminación a hacia los pacientes que sufren patologías mentales. Por ejemplo, el seguro catastrófico de las Isapres en Chile excluye dentro de su cobertura todas las hospitalizaciones y tratamientos de patologías psiquiátricas ${ }^{43}$. A su vez, los planes de las Isapres presentan en las consultas y tratamiento de psiquiatría y psicología una cobertura notablemente inferior que las enfermedades físicas.

El caso de los Estados Unidos de Norteamérica refleja un intento por combatir la inequidad entre la salud mental y física. En 2008 se promulgó el "Acta de Paridad de la Salud Mental y Equidad de la Adicción", con la cual se estableció un mínimo nacional de paridad, determinando que la salud física y la salud mental deben contar con condiciones similares de financiamiento y acceso a los tratamientos ${ }^{44}$. Esta ley federal se basó en el hecho de que para casi todos los trastornos mentales se han desarrollado métodos de diagnóstico fiables al igual que tratamientos y servicios seguros, eficaces y costo-efectivos $^{10,45}$. A su vez, los estados que previamente contaban con leyes de paridad disminuyeron considerablemente la carga financiera de las familias con hijos que presentan enfermedades mentales, contribuyendo, por ejemplo, a que los padres puedan mantener un mejor cuidado de sus hijos ${ }^{46}$.

Con la aplicación en Estados Unidos de Norteamérica del Acta de 2008 y el cambio en la comprensión de la salud mental, el gasto en salud mental disminuyó en relación al gasto total en salud $^{47}$. Aunque aún no hay evidencia que muestre una disminución en la incidencia de los trastornos mentales, se espera que el aumento de la cobertura y beneficios de atención de salud mental reduzca la ocurrencia de este tipo de trastornos, las tasas de suicidio, abuso de alcohol y delincuencia juvenil ${ }^{48}$.

\section{Discusión}

Las condiciones neuropsiquiátricas corresponden a casi un cuarto del total de años de vida saludables perdidos por vivir con discapacidad 
(AVISA) en Chile y diversas investigaciones han demostrado que el tratamiento mental puede ser costo-efectivo. Sin embargo, si se compara con países de ingresos similares, Chile presenta un importante déficit en el financiamiento de la salud mental. El porcentaje actual del presupuesto público de salud destinado a salud mental es muy inferior al esperado para el año 2010, y la mitad de las patologías mentales presentadas como prioritarias en 2000 hoy no tienen un tratamiento asegurado a través del AUGE/GES.

Considerando que en la última Estrategia Nacional de Salud (2011-2020) se declara la intención de dar a la salud mental una atención digna y similar a la atención de salud física, proponemos las siguientes medidas:

1) Destinar un mayor porcentaje del presupuesto público de salud a salud mental, para alcanzar al menos 5\% que se había propuesto hace más de una década para el 2010 y que se acerca a $5,1 \%$ destinado por los países de altos ingre$\operatorname{sos}^{25}$. Al 2020 se podría plantear una meta de $10 \%$, la cual sería más acorde al gasto económico que significan las enfermedades mentales para el país.

2) Destinar parte del presupuesto en salud mental a investigación para la generación de evidencia sobre el caso de Chile.

3) Priorizar las patologías mentales dentro de las nuevas enfermedades que serán incorporadas al plan AUGE/GES el año 2016.

4) Promover una ley similar al Acta de Paridad de la Salud Mental y Equidad de la Adicción de Estados Unidos de Norteamérica para disminuir la discriminación que sufren las personas con trastornos mentales en Chile.

Agradecimientos: Los autores agradecen el financiamiento de CONICYT/ FONDAP/ 15110017 y CONICYT/ FONDECYT Inicio/ 11110041. A su vez, agradecen la ayuda de Catalina Letelier, María Paz Ariztía y Maureen Berho.

\section{Referencias}

1. Bertolote J, Fleischmann A. Suicide and psyquiatric diagnosis: a worldwide perspective. World Psychiatry 2002; 1 (3): 181-5.

2. Retamal P, Luego J, Trebilcock J. Epidemiología del suicidio en Chile. Acta Med CSM 2010; 4 (1): 13-21.
3. Organisation for Economic Co-operation and Development. Health at glance 2011: OECD indicators. Paris: OECD; 2011.

4. Organisation for Economic Co-operation and Development. Country statistical profile: Chile. Paris: OECD; 2013.

5. Departamento de Estadísticas e Información de la Salud. 10 primeras causas de muerte. Chile 2000-2010. Santiago: DEIS; 2010.

6. Silva D, Vicente B, Saldivia S, Kohn R. Conducta suicida y trastornos psiquiátricos en Chile, un estudio poblacional. Rev Med Chile 2013; 141 (10): 1275-82.

7. Departamento de Estadísticas e Información de la Salud. Mortalidad en Ambos Sexos de 20 a 44 Años: Según Causas Específicas de Defunción. Santiago: DEIS; 2006.

8. Departamento de Estadísticas e Información de la Salud. Mortalidad en Hombres de 20 a 44 Años: Según Causas Específicas de Defunción. Santiago: DEIS; 2006.

9. Insel T. Assessing the economic costs of serious mental illnes. Am J Psychiatry 2008; 165 (6): 663-5.

10. World Health Organization. Investing in mental health. Evidence for action. Geneva: WHO; 2013.

11. Organisation for Economic Co-operation and Development. Policy Brief: Mental Health in OECD Countries. OECD Observer; 2008.

12. Organización Mundial de la Salud. Proyecto de plan de acción integral sobre Salud Mental 2013-2020. 66a Asamblea Mundial de la Salud; 2013.

13. Ministerio de Salud de Chile. Informe Final. Estudio de Carga por Enfermedad y Carga Atribuible. Santiago: MINSAL; 2007.

14. Superintendencia de Salud, Gobierno de Chile. Análisis de Licencias Médicas y Gasto en Subsidios por Incapacidad Laboral Año 2011. Santiago: Departamento de Estudios y Desarrollo, 2013.

15. Valdés C, Errázuriz P. Salud Mental en Chile: El Pariente Pobre del Sistema de Salud. Serie Claves de Políticas Públicas, Instituto de Políticas Públicas Universidad Diego Portales. 2012.

16. Ministerio de Salud de Chile. Plan Nacional de Salud Mental y Psiquiatría. Santiago: MINSAL; 2000.

17. Aprueba Garantías Explícitas en Salud del Régimen General de Garantías en Salud. Diario Oficial de la República de Chile, nº 40.492, (5-02-2013).

18. Minoletti A, Zaccaria A. Plan Nacional de Salud Mental en Chile: 10 años de experiencia. Rev Panam Salud Publica 2005; 18 (4/5): 346-58.

19. Vicente B, Rioseco P, Saldivia S, Kohn R, Torres S. Estudio Chileno de Prevalencia de Patología Psiquiátrica (DSM-III-R/CIDI) (ECPP). Rev Med Chile 2002; 130: 527-36. 
20. Inostroza M. Malas noticias para el plan AUGE. Rev Chil Cir 2013; 65 (4): 297-8.

21. Ministerio de Salud de Chile. Estrategia Nacional de Salud para el Cumplimiento de los Objetivos Sanitarios de la Década 2011-2020. Santiago: MINSAL; 2011.

22. HM Government, Department of Health. No Health Without Mental Health: A cross-government mental health outcomes strategy for people of all ages. London: HM Government; 2011.

23. Department of Human Services. Because Mental Health Matters: Victorian Mental Health Reform Strategy 2009 -2019. Australia: Mental Health and Drugs Division, Department of Human Services; 2009.

24. Organización Mundial de la Salud, Ministerio de Salud de Chile. Informe WHO-AIMS sobre Sistema de Salud Mental en Chile. Santiago: OMS, MINSAL; 2006.

25. Universidad de Chile. Sistema de salud mental en Chile. Santiago: WHO, PAHO, MINSAL; 2014.

26. Ministerio de Salud de Chile. Encuesta Nacional de Salud [ENS] 2003. Santiago: MINSAL; 2004.

27. Ministerio de Salud de Chile. Resultados Encuesta Nacional de Salud [ENS] 2009-2010. Santiago: MINSAL; 2011.

28. Departamento de Estadísticas e Información de la Salud. Mortalidad por Causa, según Sexo. Chile 2000 - 2009. Santiago: DEIS; 2010.

29. Gibbons R, Hur K, Bhaumik D, Mann J. The relationship between antidepressant medication use and rate of suicide. Arch Gen Psychiatry 2005; 62: 165-72.

30. Lund C, De Silva M, Plagerson S, Cooper S, Chisholm $\mathrm{D}$, Das J, et al. Poverty and mental disorders: breaking the cycle in low-income and middle-income countries. Lancet 2011; 378 (9801): 1502-14.

31. Berto P, D’Ilario D, Ruffo P, Di Virgilio R, Rizzo F. Depression: Cost-of-illness Studies in the International Literature, a Review. J Mental Health Policy Econ 2000; 3: 3-10.

32. Hu T. Perspectives: an international review of the national cost estimates of mental illness, 1990-2003. J Mental Health Policy 2006; 9 (1): 3-13.

33. Mihalopoulos C, Vos T, Pirkis J, Carter R. The Economic Analysis of Prevention in Mental Health Programs. Annu Rev Clin Psycho 2011; 7: 169-201.

34. Ministerio de Salud de Chile. Guía Clínica Auge. Depre- sión en personas de 15 años y más. Santiago: MINSAL; 2013.

35. Vöhringer $P$, Jiménez $M$, Igor $M$, Fores $G$, Correa $M$, Sullivan $\mathrm{M}$, et al. A clinical predictive score for mood disorder risk in low income primary care settings. Journal of Affective Disorders 2013; 151: 1125-31.

36. Mezuk B, Eaton W, Albrecht S, Golden S. Depression and Type 2 Diabetes Over the Lifespan: A meta-analysis. Diabetes Care 2008; 31 (12): 2383-90.

37. Andersen K, Lolk A, Kragh-Sørensen P, Petersen N, Green A. Depression and the Risk of Alzheimer Disease. Epidemiology 2005; 16 (2): 233-8.

38. Ziegelstein R. Depression in Patients Recovering from a Myocardial Infarction. JAMA 2001; 286 (13): 1621-7.

39. National Business Group on Health. An Employer's Guide to Behavioral Health Services. Washington, DC: Center for Prevention and Health Services; 2005.

40. World Health Organization. The World Health Report 2001: Mental Health - New Understanding, New Hope. Ginebra: WHO; 2001.

41. Knapp M, McDaid D, Mossialos E, Thornicroft G. Salud mental en Europa: políticas y práctica. Líneas futuras en salud mental. España: Observatorio Europeo de Políticas y Sistemas Sanitarios; 2007.

42. Mental Health Promotion and Mental Illness Prevention: The Economic Case. London: Departament of Health; 2011.

43. Paleo D. Cómo y cuándo usar la cobertura catastrófica adicional de la isapre. El Mercurio 2013; Sect. B.

44. Fowler W, Saturday O. Mental Health Parity. Lexington: The Council of State Governments, 2009.

45. Frank R, Goldman H, McGuire T. Will parity in coverage result in better mental health care? $\mathrm{N}$ Engl J Med 2001; 345 (23): 1701-4.

46. Demchak C. Financial Relief: The Effect of State Mental Health Parity Laws on Families of Children with Mental Health Care Needs. Find Brief 2007; 10 (6): 1-3.

47. Aggarwal N, Rowe M. The Individual Mandate, Mental Health Parity, and the Obama Health Plan. Adm Policy Ment Health 2013; 40 (4): 255-7.

48. García R. Equity for all? Potential impact of the Mental Health Parity and Addiction Act of 2008. J Leg Med 2010; 31 (1): 137-55. 